\title{
Multivariable Regression Analysis of Clinical Data from the Randomized-Controlled EffPac Trial: Efficacy of Femoropopliteal Drug-Coated Balloon Angioplasty
}

\author{
Selma Mietz ${ }^{1} \cdot$ Thomas Lehmann $^{2} \cdot$ Ulf Teichgräber $^{1,3}$
}

Received: 29 November 2019/Accepted: 12 March 2020/Published online: 1 April 2020

(C) The Author(s) 2020

\begin{abstract}
Purpose The post-hoc multivariable analysis of EffPac study data aimed to identify explanatory variables for efficacy of femoropopliteal artery angioplasty.

Methods In the prospective, randomized, controlled EffPac study, patients were allocated to either DCB or plain old balloon angioplasty. Multivariable regression including interaction analysis was conducted to assess the impact of selected variables on the outcome measures of late lumen loss (LLL) at 6 months, and on binary restenosis, target lesion revascularization (TLR), clinical improvement, and hemodynamic improvement at 12 months.

Results A total of 171 patients ( $69 \pm 8$ years, 111 men) were treated at 11 German centers. Hypertension increased, and advanced age decreased LLL $(B$ coefficient $[B]: 0.7$ [95\% CI -0.04 to 1.3 ], $p=0.06$ and -0.3 per 10 years [95\% CI -0.5 to 0.01$], p=0.06$, respectively). DCB angioplasty decreased odds of 12-month TLR and binary restenosis (OR 0.4 [95\% CI 0.2 to 0.8 ], $p=0.01$ and OR 0.1 [ $95 \%$ CI 0.01 to 0.6], $p=0.02$, respectively). Lesion length and severe calcification decreased clinical
\end{abstract}

Electronic supplementary material The online version of this article (https://doi.org/10.1007/s00270-020-02452-2) contains supplementary material, which is available to authorized users.

Ulf Teichgräber

ulf.teichgraeber@med.uni-jena.de

1 Department of Radiology, Jena University Hospital, Jena, Germany

2 Center for Clinical Studies, Jena University Hospital, Jena, Germany

3 Institut für Diagnostische und Interventionelle Radiologie, Universitätsklinikum Jena, Am Klinikum 1, 07747 Jena, Germany improvement $(B:-0.1$ per $10 \mathrm{~mm}[95 \% \mathrm{CI}-0.1$ to -0.03 ], $p=0.001$ and -0.1 [95\% CI -1.7 to -0.1 ], $p=0.03$, respectively). DCB angioplasty in former smokers improved ABI (0.2 [95\% CI 0.01 to 0.5 ], $p=0.04)$. Conclusion DCB angioplasty decreased the incidence of 12-month restenosis and TLR. Increasing lesion length and severe calcification reduced clinical improvement. Hypertension is suspected to facilitate, and advanced age to mitigate LLL. DCB improved ABI most in former smokers.

Keywords Drug-coated balloon angioplasty . Femoropopliteal lesions · Hypertension · Regression analysis

\section{Introduction}

Numerous randomized trials on femoropopliteal artery disease demonstrated that drug-coated balloon (DCB) angioplasty effectively decreased late lumen loss (LLL) [1-9] and increased primary patency [10-14]. However, heterogeneity among studies is substantial: The mean number of patients needed to treat within 1 year to prevent a single target lesion revascularization (TLR) ranges between 4 and 33 [15].

Different effect sizes of DCB across studies mainly originate from DCB types or study-specific procedure details, whereas effect size within studies may depend on patient and lesion characteristics. For instance, Albrecht et al. reported on greater LLL and Laird et al. found a lower incidence of primary patency with increasing lesion 
length after both DCB and plain old balloon angioplasty (POBA) $[16,17]$. Scheinert et al. found lesion length, total occlusion, and critical limb ischemia to be associated with 12-month TLR after DCB [18]. Fanelli et al. described a worse impact of severe calcification on LLL and primary patency after DCB angioplasty [19], and severe dissections were identified as risk factor for restenosis after POBA [20, 21].

The randomized EffPac trial revealed a smaller 6-month LLL (primary endpoint), a higher incidence of 12-month primary patency, and a lower risk of 12-month TLR after DCB angioplasty compared to POBA [22]. The purpose of this post-hoc interaction and multivariable analysis of EffPac trial data was to assess interaction effects of selected explanatory variables with treatment, and to identify predictors of morphologic, clinical, and hemodynamic outcome measures after femoropopliteal angioplasty.

\section{Methods}

\section{Study Design, Patients, and Procedure}

Consecutive patients with symptomatic, atherosclerotic, femoropopliteal stenosis, or occlusion $\leq 150 \mathrm{~mm}$ were included in the prospective, randomized, controlled trial at 11 German sites between September 2015 and December 2016. Patients were allocated 1:1 to either DCB or POBA and blinded to the treatment strategy. Successful pre-dilation for at least $30 \mathrm{~s}$ without flow-limiting dissection or relevant residual stenosis was a precondition to inclusion. Detailed eligibility criteria are available (online resource 1, Table A1).

Investigators were not blinded to the study device. The investigational DCB (Luminor-35 paclitaxel-coated balloon, iVascular S.L.U., Barcelona, Spain) was used according to manufacturer's instruction. Inflation of DCB or control device (standard balloon) should have lasted $60 \pm 10 \mathrm{~s}$ up to the nominal pressure. Bailout stenting in case of flow-limiting dissection despite prolonged inflation was permitted. The angiographic and Doppler sonographic results were evaluated by an independent core laboratory.

Detailed description of device and procedure, and the full study protocol had been provided earlier [22, 23]. The EffPac trial is registered with ClinicalTrials.gov (NCT02540018).

\section{Study Outcome Measures}

Primary endpoint of the EffPac trial was 6-month LLL. Outcomes measured of this post-hoc analysis were impact of baseline patient, lesion, and procedure characteristics on 6-month LLL, binary restenosis, TLR, improvement in Rutherford-Becker category (clinical improvement), and improvement in ankle-brachial index (ABI) (hemodynamic improvement) at 12 months. Outcome measures were specified as unstandardized regression coefficient $B(B)$ in case of LLL, Rutherford-Becker category, and ABI or as odds ratio (OR) in case of binary restenosis and TLR. LLL was defined as difference between minimum lumen diameter immediately after angioplasty and at follow-up. Restenosis occurred with $>50 \%$ diameter stenosis of the target lesion by angiography or peak systolic velocity ratio of $\geq 2.5$ by duplex ultrasound. Detailed description of outcome measures is available at online resource 1 .

\section{Statistical Analysis}

Post-hoc multivariable regression analysis was conducted in three steps separately for each outcome measure. In the first step, 15 independent variables (age, sex, hypertension, body mass index, diabetes mellitus, smoking status, percentage diameter stenosis, lesion length, calcification, total occlusion, bailout stenting, dissection, Rutherford-Becker category, ABI, Transatlantic Inter-Society Consensus for the Management of Peripheral Arterial Disease classification (TASC) II classification) were subjected to formal interaction analysis with treatment. In case of a $p$ value for interaction of $<0.1$, variables concerned, and their related interaction terms were included in the final multivariable analysis (online resource 1, Table A2). In the second step, variables that showed a $p$ value for interaction of $\geq 0.1$ were subjected to univariable analysis (linear or logistic regression). Variables with a $p$ value of $<0.1$ for univariable association with the respective outcome were included in the final multivariable analysis (online resource 1 , Table A3). In the third step, all variables with $p<0.1$ at step 1 or 2 , and the variable of treatment were included in the final model. Additionally, box plots and Spearman's correlation were provided to visualize descriptive associations of clinically relevant variables to LLL (lesions with bailout stenting were excluded) and hemodynamic improvement. Continuous variables were expressed as mean \pm standard deviation, and differences were assessed by Mann-Whitney $U$ test. Categorical variables were reported as count and percentage, and differences were assessed by Fisher's exact test. Statistical analysis was performed using SPSS Statistics 25.0 (IBM, Armonk, NY, USA). 


\section{Results}

\section{Study Population}

Baseline patient, lesion, and procedure characteristics were well matched across treatment groups (Table 1). Six-month LLL was assessed in 53 of 85 (62.4\%) DCB and in 63 of 86 (73.3\%) POBA patients. One-year follow-up was completed in 76 (89.4\%) DCB and 76 (88.4\%) POBA patients. Outcomes at 6 and 12 months were published previously
[22]. In short, DCB proved to be effective regarding 6-month LLL, 12-month primary patency, and TLR.

\section{Predictors of Morphologic Outcome Measures}

\section{Late Lumen Loss}

For the joint effect of CTO and treatment on LLL, a $p$ value of 0.06 was determined, and thus, according to study protocol, CTO and its corresponding interaction term were
Table 1 Patient, lesion, and procedure characteristics

\begin{tabular}{|c|c|c|c|c|}
\hline Characteristics & Total $(n=171)$ & $\mathrm{DCB}(n=85)$ & POBA $(n=86)$ & $p$ value \\
\hline Age, year & $68.1 \pm 8.2$ & $68.0 \pm 7.5$ & $68.1 \pm 8.8$ & $p=0.96$ \\
\hline Male & $111(64.9)$ & $51(60.0)$ & $60(69.8)$ & $p=0.20$ \\
\hline Body mass index ${ }^{a}$ & $27.5 \pm 4.7$ & $27.4 \pm 4.8$ & $27.7 \pm 4.7$ & $p=0.57$ \\
\hline Underweight & $4(2.4)$ & $1(1.2)$ & $3(3.6)$ & \\
\hline Normal weight & $50(30.5)$ & $30(37.0)$ & $20(24.1)$ & \\
\hline Pre-obesity & $68(41.5)$ & $28(34.6)$ & $40(48.2)$ & \\
\hline Obesity & $42(25.6)$ & $22(27.2)$ & $20(24.1)$ & \\
\hline Smoking status & & & & $p=0.94$ \\
\hline Current smoker & $71(41.8)$ & $34(40.5)$ & $37(43.0)$ & \\
\hline Former smoker & $71(41.8)$ & $36(42.9)$ & $35(40.7)$ & \\
\hline Never smoked & $28(16.5)$ & $14(16.7)$ & $14(16.3)$ & \\
\hline Diabetes mellitus & $66(38.6)$ & $31(36.5)$ & $35(40.7)$ & $p=0.64$ \\
\hline Hypertension & $147(86.0)$ & $74(87.1)$ & $73(84.9)$ & $p=0.83$ \\
\hline Hyperlipidemia & $119(69.6)$ & $60(70.6)$ & $59(68.6)$ & $p>0.99$ \\
\hline Rutherford category & & & & $p=0.53$ \\
\hline 2 & $31(18.2)$ & $13(15.3)$ & $18(21.2)$ & \\
\hline 3 & $135(79.4)$ & $69(81.2)$ & $66(77.6)$ & \\
\hline 4 & $3(1.8)$ & $2(2.4)$ & $1(1.2)$ & \\
\hline 5 & $1(0.6)$ & $1(1.2)$ & 0 & \\
\hline Ankle-brachial index & $0.74 \pm 0.23$ & $0.73 \pm 0.23$ & $0.74 \pm 0.23$ & $p=0.78$ \\
\hline Lesion length, mm & $57.4 \pm 41.2$ & $59.1 \pm 43.4$ & $55.8 \pm 39.1$ & $p=0.60$ \\
\hline Total occlusion & 39 (22.9) & $17(20.2)$ & $22(25.6)$ & $p=0.47$ \\
\hline Diameter stenosis, \% & $89.1 \pm 9.3$ & $88.0 \pm 9.8$ & $90.1 \pm 8.8$ & $p=0.16$ \\
\hline $\mathrm{TASC}_{\mathrm{II}}^{\mathrm{b}}$ & & & & $p=0.75$ \\
\hline A & $113(66.1)$ & $55(64.7)$ & $58(67.4)$ & \\
\hline $\mathrm{B}$ & $58(33.9)$ & $30(35.3)$ & $28(32.6)$ & \\
\hline Calcification & & & & $p=0.11$ \\
\hline None/mild & $83(49.1)$ & $45(54.2)$ & $38(44.2)$ & \\
\hline Moderate & $73(43.2)$ & $35(42.2)$ & $38(44.2)$ & \\
\hline Severe & $13(7.7)$ & $3(3.6)$ & 10 (11.6) & \\
\hline Dissection & $67(39.2)$ & $32(37.6)$ & $35(40.7)$ & $p=0.76$ \\
\hline Bailout stenting & $29(17.1)$ & $13(15.3)$ & $16(18.8)$ & $p=0.68$ \\
\hline
\end{tabular}

$\overline{\text { Categorical values are presented as counts (percentages); continuous values are presented as mean } \pm \mathrm{s}-}$ tandard deviation

$D C B$ drug-coated balloon, $P O B A$ plain old balloon angioplasty

${ }^{a}$ Body mass index $\left(\mathrm{kg} / \mathrm{m}^{2}\right)$ according to the World Health Organization (WHO) definition: normal weight 18.5-24.9, pre-obesity 25.0-29.9, obesity $\geq 30$

${ }^{\mathrm{b}}$ Inter-Society Consensus for the Management of Peripheral Arterial Disease classification 
included in the multivariable model (online resource 1, Table A2). Finally, multivariable analysis did not reveal a simultaneous impact of CTO and treatment on LLL (Fig. 1).

Treatment Multivariable regression revealed a trend to decrease LLL with DCB compared to POBA ( $B$ coefficient $[B]:-0.8$ [95\% CI -2.0 to 0.4$], p=0.17$ ) (Fig. 1A). This finding was supported by results from descriptive statistics that found less LLL after DCB versus POBA irrespective of whether or not arteries were occluded, dissected, moderately or severely calcified, or whether patients had hypertension (Fig. 2A-D).

Lesion complexity Total occlusion, dissection, and calcification did not affect LLL in both treatment groups (Fig. 2A-C). However, with DCB there was a weak negative correlation between LLL and lesion length (DCB: $r_{s}=-0.18, \quad p=0.25 ; \quad$ POBA: $\left.r_{s}=0.16, \quad p=0.24\right)$. Therefore, benefit of DCB tended to rise with increasing lesion length (Fig. 3A).

Hypertension Hypertension tended to increase LLL (B: 0.7 [95\% CI -0.04 to 1.3$], p=0.06$ ) (Fig. 1). The impact was more apparent in the DCB than in the POBA group (0.4 vs. $-0.3 \mathrm{~mm}, p=0.09$ and 1.3 vs. $1.1 \mathrm{~mm}, p=0.70$, respectively) (Fig. 2D).

Age Higher age tended to decrease LLL (B: $-0.3[95 \%$ CI -0.5 to 0.01$], p=0.06$ ) (Fig. 1A). A weak negative correlation was visible with POBA $\left(\mathrm{r}_{\mathrm{s}}=-0.24, p=0.09\right)$. Thus, the benefit of DCB over POBA tended to decrease with advanced age (Fig. 3B).

Body mass index No correlation was seen between LLL and BMI $\left(r_{s}=0.01, p=0.94\right.$ with $\mathrm{DCB}, r_{s}=0.08$, $p=0.59$ with POBA) (Fig. 3C).

\section{Binary Restenosis}

Six-month luminal gain was associated with less binary restenosis than luminal loss ( $0 \%$ vs. $33.8 \%, p=0.001$, Table 2). None of the selected variables interacted with the treatment effect on binary restenosis (online resource 1, Table A2). Multivariable analysis revealed that DCB angioplasty independently reduced the odds of binary restenosis (OR 0.4 [95\% CI 0.2 to 0.8 ], $p=0.01$ ) (Fig. 4A).

In the POBA, but not in the DCB group, total occlusions tended to be more frequently restenosed (POBA: $52.9 \%$ vs. $29.5 \%, p=0.08$ vs. $\mathrm{DCB}: 8.3 \%$ vs. $12.0 \%, p>0.99)$ (Fig. 4B).

\section{Predictors of Clinical Outcome Measures}

Target lesion revascularization Luminal gain at 6 months was associated with less 12-month TLR than luminal loss ( $0 \%$ vs. $18.5 \%, p=0.03$, Table 2$)$. None of the selected variables interacted with the treatment effect (online resource 1, Table A2). Multivariable analysis found TLR to be less likely in patients who underwent DCB angioplasty (OR 0.1 [95\% CI 0.01 to 0.6], $p=0.02$ ) (Fig. 5A).

Clinical improvement Interaction analysis of nutritional status with treatment revealed a $p$ value of 0.03 (online resource 1 , Table $\mathrm{A} 2$ ), and thus, nutritional status and its corresponding interaction term were included in the final multivariable analysis. Finally, combined effect of nutritional status and treatment did not impact clinical improvement. The same applied for the variable of treatment alone, that did not affect clinical improvement $(B: 0.3$ [95\% CI -0.3 to 0.8 ] $p=0.38$ ). However, it should be noted that patients who underwent TLR were included in the assessment of Rutherford-Becker category at
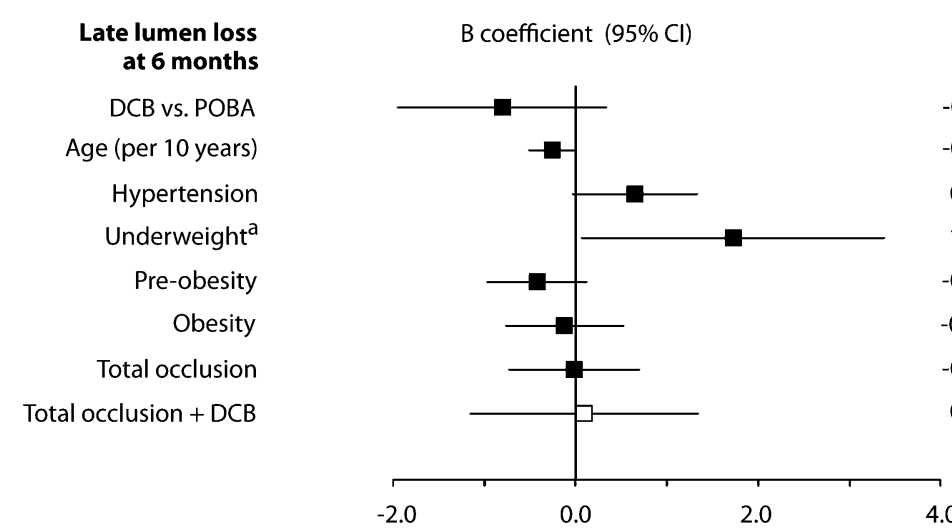

Fig. 1 Association of late lumen loss at 6 months with selected baseline and procedure variables, determined by post-hoc multivariable analysis. $B$ coefficients of single independent variables are pictured as black squares and joint effects of independent variables with treatment as white squares with their corresponding $95 \%$ confidence intervals. ${ }^{\text {a}}$ Four underweight patients were analyzed. $D C B$ drug-coated balloon, $P O B A$ plain old balloon angioplasty, TASC Inter-Society Consensus for the Management of Peripheral Arterial Disease classification 

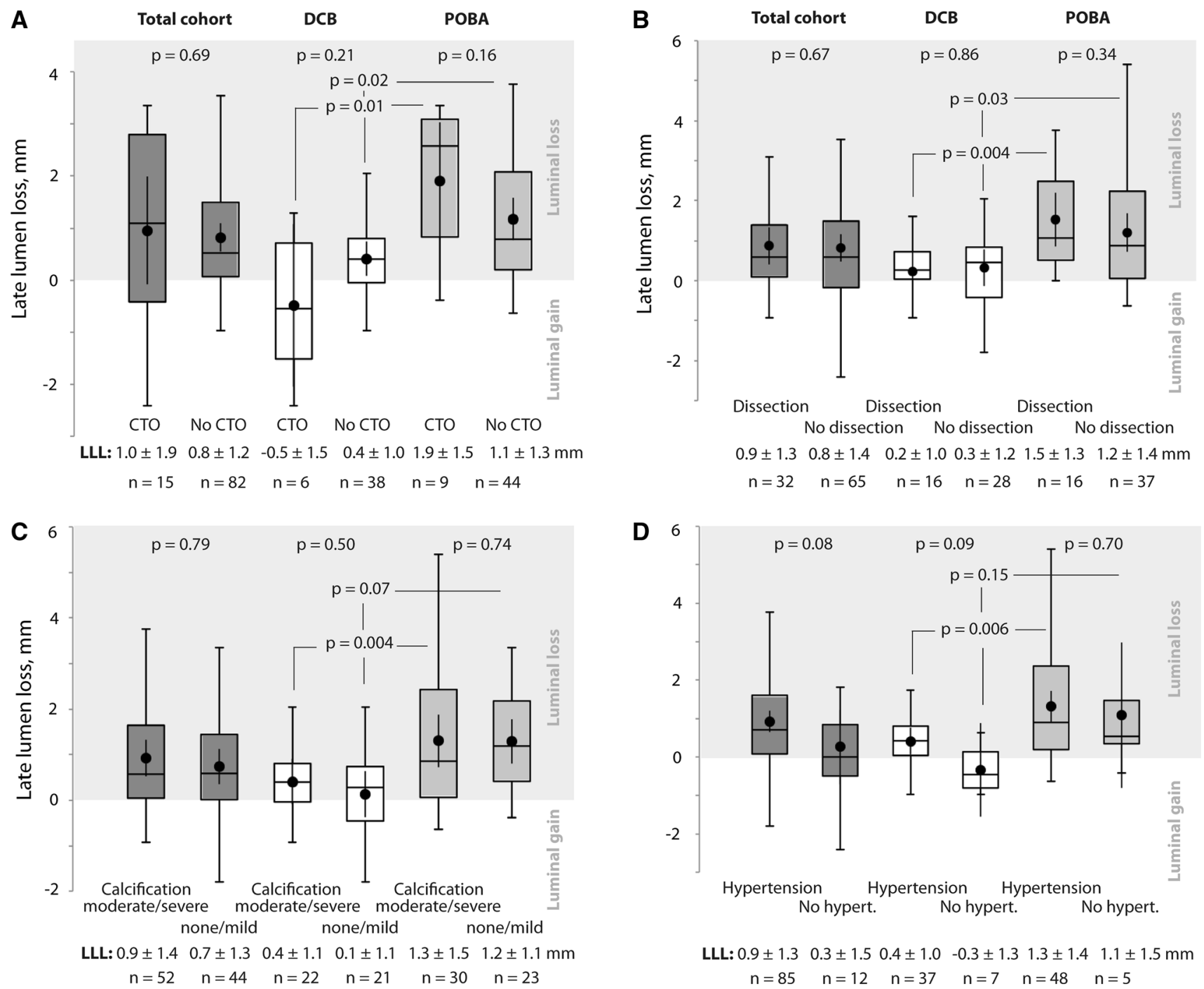

Fig. 2 Association of 6-month late lumen loss with total occlusion (A), dissection (B), moderate or severe calcification (C), and hypertension (D). Lesions that were treated with stent were excluded from this analysis. Box plots indicate median and interquartile ranges.

12 months. Lesion length and severe calcification turned out to independently decrease clinical improvement $(B$ : -0.07 per $10 \mathrm{~mm} \mathrm{[95 \%} \mathrm{CI}-0.1$ to -0.03$], p=0.001$ and $B:-0.88[95 \% \mathrm{CI}-1.7$ to -0.1$], p=0.03$, respectively). Lesions that were more severely stenosed at baseline were associated with increased clinical improvement (Fig. 5B).

\section{Predictors of Hemodynamic Improvement}

Simultaneous effect of smoking status and treatment on hemodynamic improvement revealed a $p$ value of 0.02 . Thus, smoking status and related interaction terms were included in the multivariable analysis (online resource 1, Table A2). Multivariable analysis indicated that DCB

D

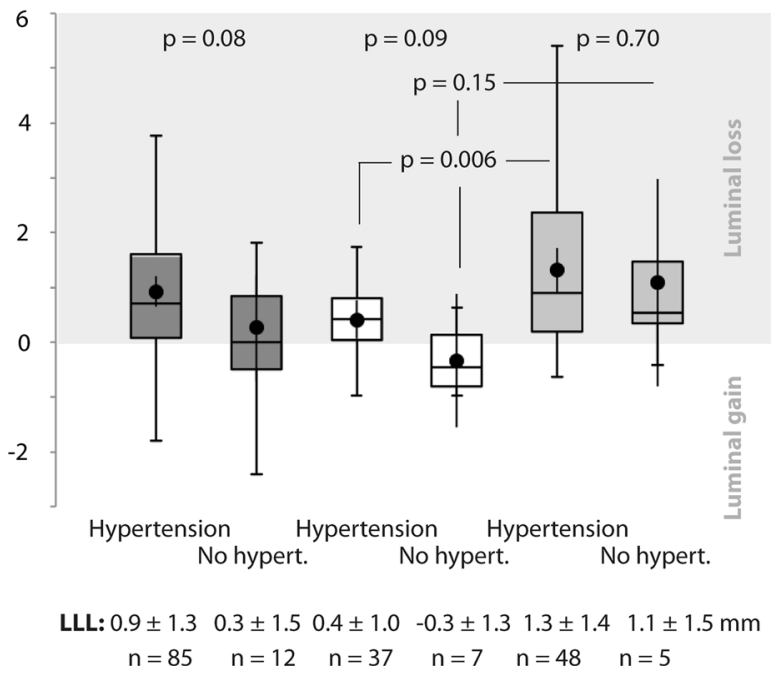

Whiskers end with the lowest and highest data point within $1.5 \times$ IQR. Dots represent means with their corresponding $95 \%$ confidence interval. $C T O$ chronic total occlusion, $D C B$ drug-coated balloon, $L L L$ late lumen loss, $P O B A$ plain old balloon angioplasty

angioplasty significantly improved hemodynamic condition in former smokers ( $B$ : 0.2 [95\% CI 0.01 to 0.48 ], $p=0.04$ ). $\mathrm{ABI}$ improvement did not differ between treatment groups in general (DCB angioplasty vs. POBA: $B:-0.13$ [95\% CI 0.02 to 0.28$], p=0.20$ ). However, patients who underwent TLR were included in 12-month ABI assessment. A higher ABI at baseline was associated with less clinical improvement (Fig. 6).

\section{Discussion}

DCB proved to be superior to POBA in both, prevention of restenosis and TLR. Lesion length and severe calcification were identified as predictors of less clinical improvement. 

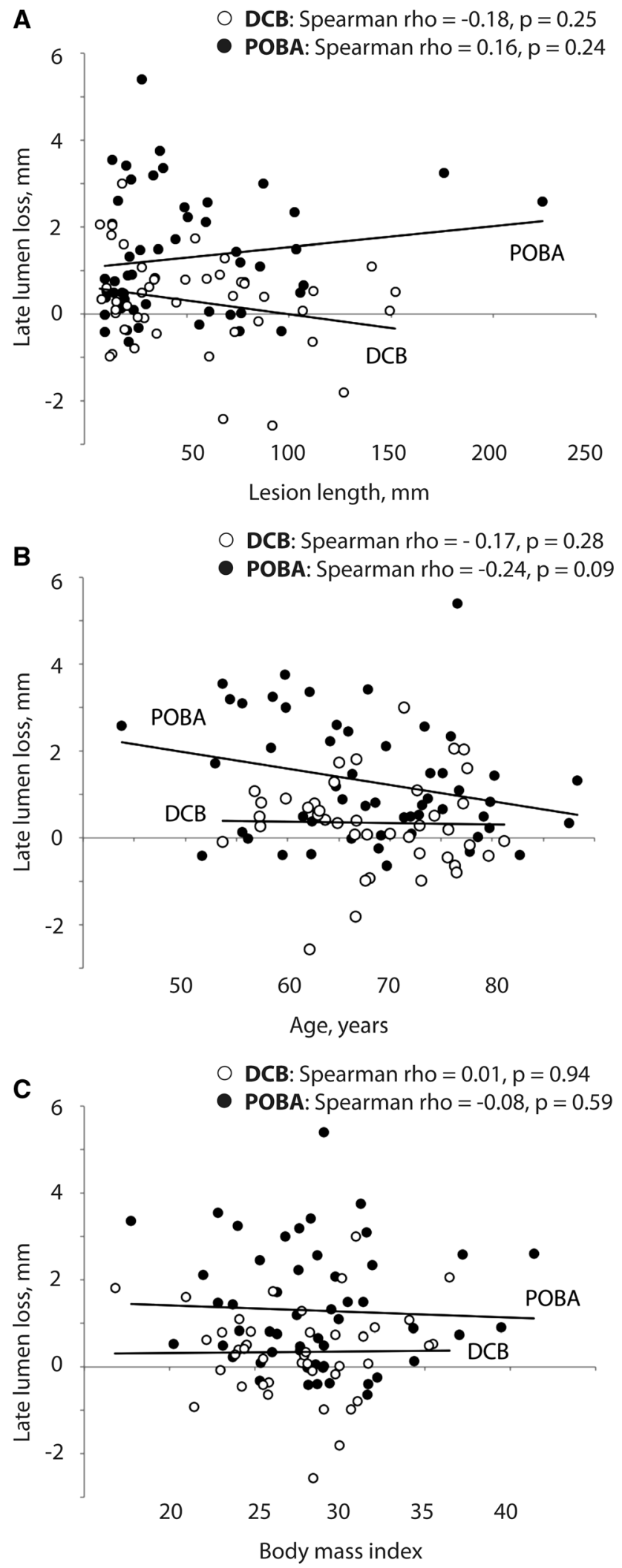

Fig. 3 Association of 6-month late lumen loss with lesion length (A), age $(\mathbf{B})$, and body mass index $(\mathbf{C})$ Lesions that were treated with stent were excluded from this analysis. $D C B$ drug-coated balloon, $P O B A$ plain old balloon angioplasty
DCB angioplasty decreased 6-month LLL more effectively than POBA, irrespective of whether or not arteries were occluded, calcified, or dissected. Our study revealed a series of hypothesis generating results on LLL. First, increasing lesion length tended to increase the benefit of DCB over POBA; second, hypertension tended to increase LLL, and third, advanced age tended to decrease LLL. No correlation was seen between LLL and BMI. Former smoker emerged as predictors of increased hemodynamic improvement after DCB. Sex, diabetes, TASC II classification, and bailout stenting did not predict outcomes.

\section{Complex Lesions}

In contrast to previous studies, in our study, increasing lesion length was not correlated with LLL [16-18]. However, the outcome of LLL only describes the quantity of loss in diameter, but not the total length of relevant luminal loss. Thus, even with similar LLL, longer lesions may have worse outcomes with respect to binary restenosis or clinical improvement, as observed in this study. The tendency of growing benefit of DCB with increasing lesion length was possibly due to chance. A dose-response relationship with paclitaxel in longer lesions might be considered.

The slight tendency of increased 6-month LLL in total occlusions treated with POBA is encouraged by findings on 12-month binary restenosis. Although calcium is suspected to impede paclitaxel penetration into the vessel wall [19], our study could not detect any disadvantage of moderately or severely calcified lesions regarding LLL in the DCB group. This may be explained by the assessment of calcification that was done only by visual estimate. Another explanation might be that in case of severe calcification, pre-dilation sufficiently loosened the calcium barrier. Finally, investigators might have positioned DCB not directly at circumferentially calcified segments of the lesion. Additional stent implantation might have supported balloon angioplasty. Despite no impact of calcification on LLL, severe calcification predicted less clinical improvement. This might be attributable to more advanced stages of disease and involvement of infrapopliteal arteries in patients with severe calcification.

Dissected lesions that were not stented did not differ in the magnitude of LLL from not dissected lesions in both treatment groups. Superiority of DCB over POBA was proven for both, dissected and not dissected lesions. However, stented dissections were excluded from analysis on LLL. Thus, our finding does not contradict previous studies that reported on increased restenosis rates after severe, but not after mild dissection [20, 21]. In our study, neither dissection nor bailout stenting predicted restenosis.

Clinical improvement increased with higher percentage diameter stenosis, and hemodynamic improvement 
Table 2 Association of late luminal loss at 6 months with morphologic and clinical outcomes at 12 months

\begin{tabular}{llll}
\hline Outcomes & Luminal loss at 6 months & Luminal gain at 6 months & $p$ value \\
\hline Binary restenosis & $24 / 71(33.8)$ & $0 / 23(0.0)$ & $p=0.001$ \\
Target lesion revascularization & $12 / 65(18.5)$ & $0 / 21(0.0)$ & $p=0.03$ \\
\hline
\end{tabular}

Values are given as counts (percentages)

Patients who underwent bailout stenting were excluded from analysis
Fig. 4 Association of 12-month binary restenosis with selected baseline and procedure variables, determined by posthoc multivariable analysis (A), and association of 12-month binary restenosis with total occlusion, excluding bailout stenting (B). $D C B$ drug-coated balloon, $P O B A$ plain old balloon angioplasty, TASC Inter-Society Consensus for the Management of Peripheral Arterial Disease classification
A

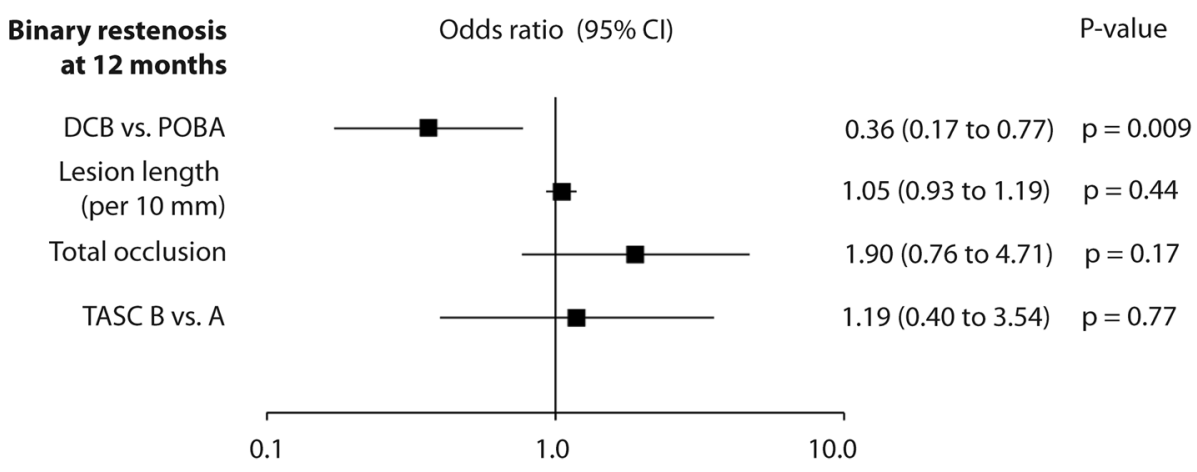

B

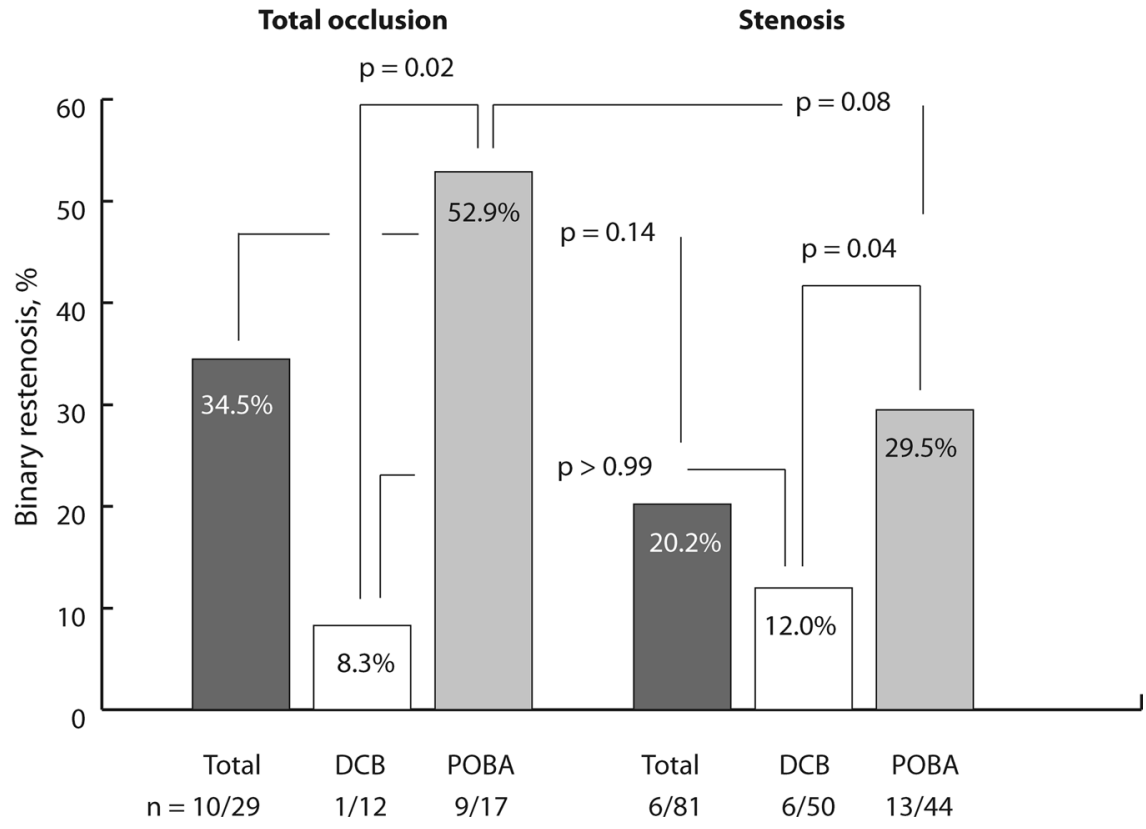

decreased with higher baseline ABI. This might be attributable to more room for improvement by revascularization in lesions with poor baseline conditions, and vice versa.

\section{Comorbidities}

The trend of decreased LLL with advanced age in the POBA group is not supported by previous studies and possibly due to chance [16, 17]. However, Han et al. reported on reduced neointimal thickness and strut coverage of coronary arteries after stent implantation in older patients [24]. This might have been due to declined endothelial function and regenerative capacity. In elderlies, synthesis of growth factors is decreased, and growth factor receptors are suppressed. This leads to an attenuated response to physical and chemical signals [25-27].

The observed tendency of an unfavorable impact of hypertension on LLL might have been due to chance. Only a few patients without hypertension were included in the analysis, and no information on control of hypertension or medication was available. Patients without a history of 
A Target lesion revascularization at 12 months

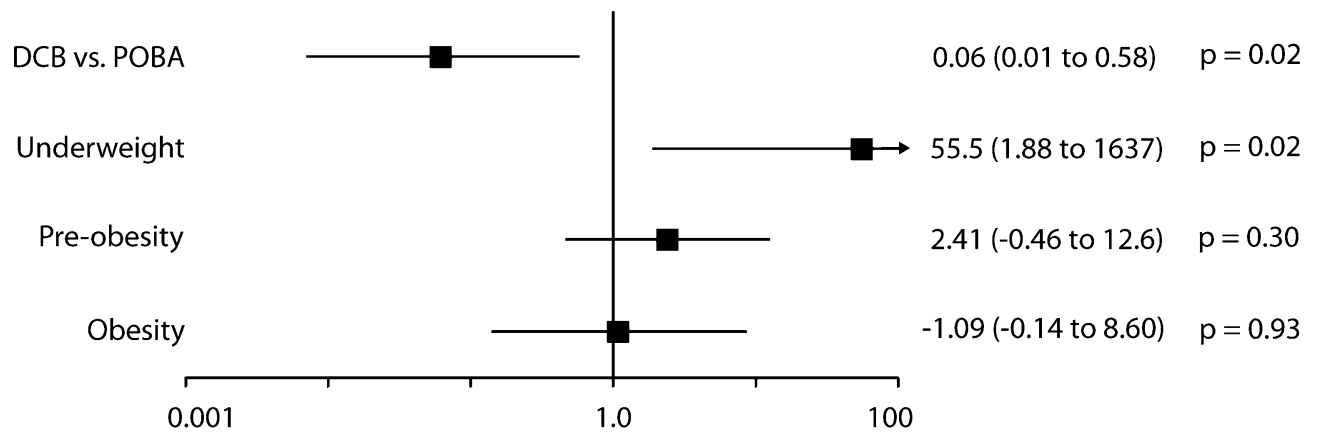

B Rutherford-Becker category Improvement at 12 months

DCB vs. POBA
Underweight
Pre-obesity
Obesity
Pre-obesity + DCB
Obesity + DCB
Diameter stenosis (per 10\%)
Lesion length (per 10 mm)
Calcification, moderate
Calcification, severe
Total occlusion
BB cailout stenting
RB category 2 (vs. 4)

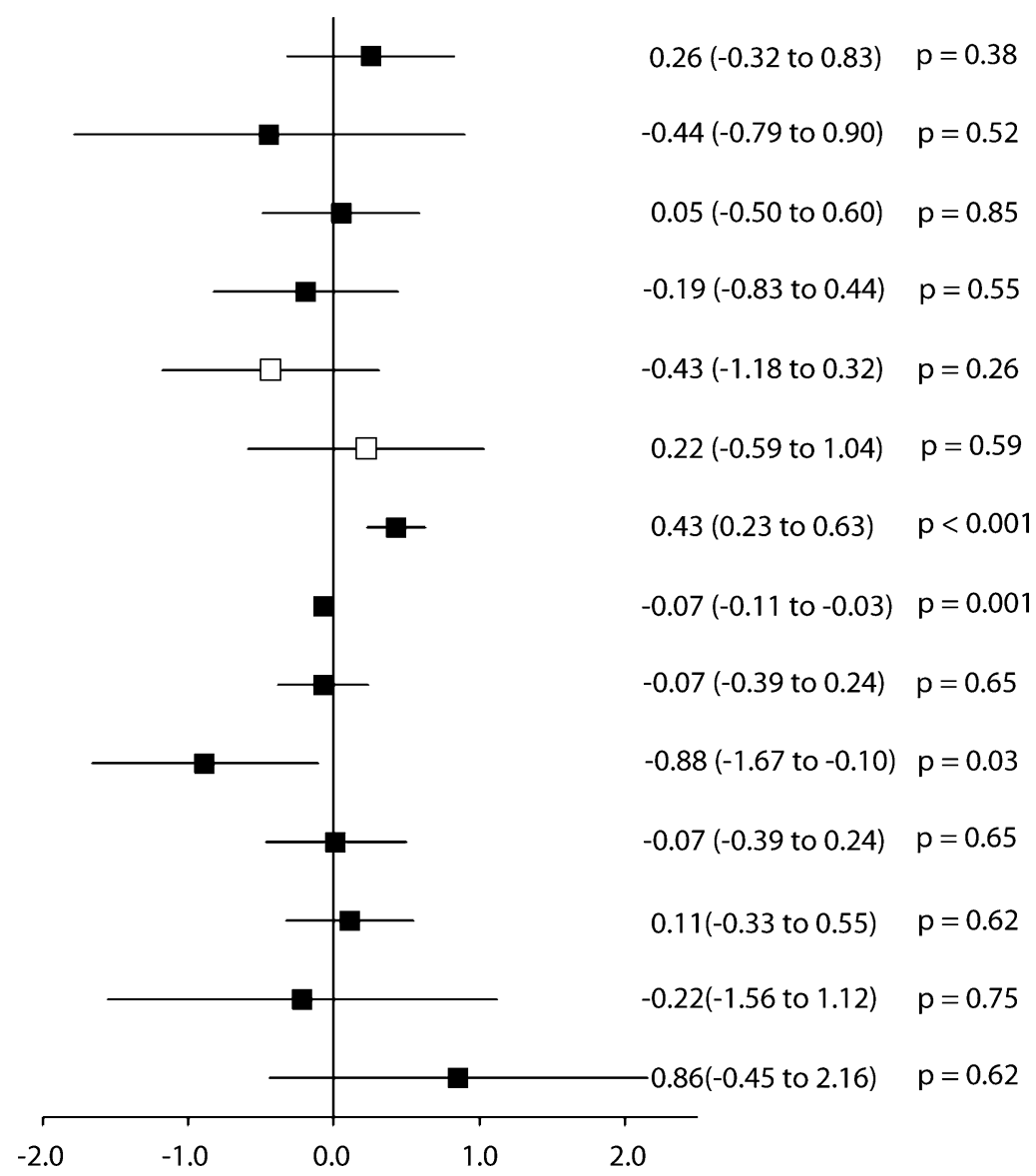

Fig. 5 Association of target lesion revascularization (A) and improvement in Rutherford-Becker category (B) at 12 months with selected baseline and procedure variables, determined by post-hoc multivariable analysis. $B$ coefficients of single independent variables are pictured as black squares and joint effects of independent variables with treatment as white squares with their corresponding 95\% confidence intervals. $D C B$ drug-coated balloon, $P O B A$ plain old balloon angioplasty, $R B$ Rutherford-Becker category hypertension might have benefited from sufficient endothelial function.

Regarding ABI, former smokers benefited most from DCB angioplasty compared to never or current smokers.
Smoking induces inflammation and modulates immune response [28]. Possibly, former smokers responded well to paclitaxel because of a still altered inflammation response 
Fig. 6 Association of improvement in ankle-brachial index $(\mathrm{ABI})$ at 12 months with selected baseline and procedure variables, determined by posthoc multivariable analysis. $B$ coefficients of single independent variables are pictured as black squares and joint effects of independent variables with treatment as white squares with their corresponding $95 \%$ confidence intervals. $D C B$ drug-coated balloon, $P O B A$ plain old balloon angioplasty, TASC Inter-Society Consensus for the Management of Peripheral Arterial Disease classification
Ankle-brachial index

Improvement at 12 months

DCB vs. POBA

Current smoker

Former smoker

Current smoker + DCB

Formert smoker + DCB

Lesion length (per $10 \mathrm{~mm}$ )

Calcification, moderate

Calcification, severe

Total occlusion
B coefficient $(95 \% \mathrm{Cl})$

P-value

Ankle-brachial index

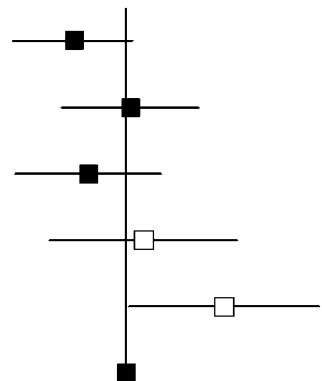

$-0.13(0.02$ to 0.28$)$

$p=0.20$

$0.01(-0.16$ to 0.18$)$

$p=0.90$

$-0.09(-0.27$ to 0.09$)$

$p=0.32$

$0.04(-0.19$ to 0.28$) \quad p=0.72$

$0.24(0.01$ to 0.48$) \quad p=0.04$

$0.002(-0.01$ to 0.01$) \quad p=0.70$

$0.05(-0.03$ to -0.14$) \quad p=0.23$

$0.02(-0.18$ to 0.22$) \quad p=0.84$

$-0.02(-0.13$ to 0.10$) \quad p=0.77$

$-0.70(-0.90$ to -0.50$) \quad p<0.001$ from previous smoking together with an already restored vascular function from smoking cessation.

\section{Limitations}

Our study was a post-hoc evaluation of prospectively achieved EffPac trial data. The EffPac trial was designated neither to investigate associations of explanatory variables with clinical outcomes nor to determine interaction effects. Thus, power and evidence are limited and findings on age, hypertension, and smoking status should be considered hypothesis generating. Study device was the Luminor-35 DCB, and thus, results may not apply equally for all DCB types.

\section{Conclusion}

Treatment strategy of DCB or POBA in femoropopliteal lesions proved to independently affect incidence of restenosis and TLR. Sex, diabetes, total occlusion, dissection, bailout stenting, baseline Rutherford category, and TASC II classification were not found to impact outcome measures. Increased lesion length and severe calcification independently decreased clinical improvement. In addition, our study revealed a trend for age to decrease and for hypertension to increase LLL. Most ABI improvement was achieved in former smokers who underwent DCB angioplasty. The later findings may have implications for future research.
Acknowledgements Open Access funding provided by Projekt DEAL.

Funding This study was funded by iVascular S.L.U., Barcelona, Spain, and Endoscout, Freiburg, Germany. Decision on manuscript content was exclusively reserved for the authors.

\section{Compliance with Ethical Standards}

Conflict of interest All authors declare that they have no conflict of interest.

Ethical Approval All procedures performed were in accordance with the ethical standards of the institutional and national research committee and with the 1964 Helsinki Declaration and its later amendments or comparable ethical standards.

Informed Consent Informed consent was obtained from all individual participants included in the study.

Consent for Publication For this type of study, consent for publication is not required.

Open Access This article is licensed under a Creative Commons Attribution 4.0 International License, which permits use, sharing, adaptation, distribution and reproduction in any medium or format, as long as you give appropriate credit to the original author(s) and the source, provide a link to the Creative Commons licence, and indicate if changes were made. The images or other third party material in this article are included in the article's Creative Commons licence, unless indicated otherwise in a credit line to the material. If material is not included in the article's Creative Commons licence and your intended use is not permitted by statutory regulation or exceeds the permitted use, you will need to obtain permission directly from the copyright holder. To view a copy of this licence, visit http://creativecommons. org/licenses/by/4.0/. 


\section{References}

1. Jia X, Zhang J, Zhuang B, et al. Acotec drug-coated balloon catheter: randomized, multicenter, controlled clinical study in femoropopliteal arteries: evidence from the acoart i trial. JACC Cardiovasc Interv. 2016;9(18):1941-9.

2. Scheinert D, Schulte KL, Zeller T, Lammer J, Tepe G. Paclitaxelreleasing balloon in femoropopliteal lesions using a BTHC excipient: twelve-month results from the BIOLUX P-I randomized trial. J Endovasc Ther. 2015;22(1):14-21.

3. Tepe G, Gogebakan O, Redlich U, et al. Angiographic and clinical outcomes after treatment of femoro-popliteal lesions with a novel paclitaxel-matrix-coated balloon catheter. Cardiovasc Interv Radiol. 2017;40(10):1535-44.

4. Werk M, Langner S, Reinkensmeier B, et al. Inhibition of restenosis in femoropopliteal arteries: paclitaxel-coated versus uncoated balloon: femoral paclitaxel randomized pilot trial. Circulation. 2008;118(13):1358-65.

5. Werk M, Albrecht T, Meyer DR, et al. Paclitaxel-coated balloons reduce restenosis after femoro-popliteal angioplasty: evidence from the randomized PACIFIER trial. Circ Cardiovasc Interv. 2012;5(6):831-40.

6. Bausback Y, Willfort-Ehringer A, Sievert H, et al. Six-month results from the initial randomized study of the ranger paclitaxelcoated balloon in the femoropopliteal segment. J Endovasc Ther. 2017;24(4):459-67.

7. Tepe G, Zeller T, Albrecht T, et al. Local delivery of paclitaxel to inhibit restenosis during angioplasty of the leg. N Engl J Med. 2008;358(7):689-99.

8. Ott I, Cassese S, Groha P, et al. Randomized comparison of paclitaxel-eluting balloon and stenting versus plain balloon plus stenting versus directional atherectomy for femoral artery disease (ISAR-STATH). Circulation. 2017;135(23):2218-26.

9. Scheinert D, Duda S, Zeller T, et al. The LEVANT I (Lutonix paclitaxel-coated balloon for the prevention of femoropopliteal restenosis) trial for femoropopliteal revascularization: first-inhuman randomized trial of low-dose drug-coated balloon versus uncoated balloon angioplasty. JACC Cardiovasc Interv. 2014;7(1):10-9.

10. Liistro F, Grotti S, Porto I, et al. Drug-eluting balloon in peripheral intervention for the superficial femoral artery: the DEBATE-SFA randomized trial (drug eluting balloon in peripheral intervention for the superficial femoral artery). JACC Cardiovasc Interv. 2013;6(12):1295-302.

11. Schroeder H, Werner M, Meyer DR, et al. Low-Dose paclitaxelcoated versus uncoated percutaneous transluminal balloon angioplasty for femoropopliteal peripheral artery disease: oneyear results of the illumenate european randomized clinical trial (randomized trial of a novel paclitaxel-coated percutaneous angioplasty balloon). Circulation. 2017;135(23):2227-36.

12. Krishnan P, Faries P, Niazi K, et al. Stellarex drug-coated balloon for treatment of femoropopliteal disease: twelve-month outcomes from the randomized ILLUMENATE pivotal and pharmacokinetic studies. Circulation. 2017;136(12):1102-13.

13. Tepe G, Schnorr B, Albrecht T, et al. Angioplasty of femoralpopliteal arteries with drug-coated balloons: 5-year follow-up of the THUNDER trial. JACC Cardiovasc Interv. 2015;8(1 Pt A): $102-8$
14. Rosenfield K, Jaff MR, White CJ, et al. Trial of a paclitaxelcoated balloon for femoropopliteal artery disease. N Engl J Med. 2015;373(2):145-53.

15. Klumb C, Lehmann T, Aschenbach R, Eckardt N, Teichgräber U. Benefit and risk from paclitaxel-coated balloon angioplasty for the treatment of femoropopliteal artery disease: a systematic review and meta-analysis of randomised controlled trials. EClinicalMedicine. 2019;16:42-50.

16. Albrecht T, Ukrow A, Werk M, et al. Impact of patient and lesion characteristics on drug-coated balloon angioplasty in the femoropopliteal artery: a pooled analysis of four randomized controlled multicenter trials. Cardiovasc Interv Radiol. 2019;42(4):495-504.

17. Laird JR, Schneider PA, Tepe G, et al. Durability of treatment effect using a drug-coated balloon for femoropopliteal lesions: 24-month results of IN.PACT SFA. J Am Coll Cardiol. 2015;66(21):2329-38.

18. Zeller T, Brodmann M, Micari A, et al. Drug-coated balloon treatment of femoropopliteal lesions for patients with intermittent claudication and ischemic rest pain. Circ Cardiovasc Interv. 2019;12(1):e007730.

19. Fanelli F, Cannavale A, Gazzetti M, et al. Calcium burden assessment and impact on drug-eluting balloons in peripheral arterial disease. Cardiovasc Interv Radiol. 2014;37(4):898-907.

20. Fujihara M, Takahara M, Sasaki S, et al. Angiographic dissection patterns and patency outcomes after balloon angioplasty for superficial femoral artery disease. $J$ Endovasc Ther. 2017;24(3):367-75.

21. Kobayashi N, Hirano K, Yamawaki M, et al. Simple classification and clinical outcomes of angiographic dissection after balloon angioplasty for femoropopliteal disease. J Vasc Surg. 2018;67(4):1151-8.

22. Teichgräber U, Lehmann $T$, Aschenbach $R$ et al. Efficacy and safety of a novel paclitaxel-nano-coated balloon for femoropopliteal angioplasty: 1-year results of EffPac trial. EuroInterventiom. 2019.

23. Teichgraber U, Aschenbach R, Scheinert D, et al. The effectiveness of the paclitaxel-coated Luminor(R) balloon catheter versus an uncoated balloon catheter in superficial femoral and popliteal arteries in preventing vessel restenosis or reocclusion: study protocol for a randomized controlled trial. Trials. 2016;17(1):528.

24. Han Z, Feng L, Du H, et al. Impact of Age on stent strut coverage and neointimal remodeling as assessed by optical coherence tomography. Medicine. 2015;94(50):e2246.

25. Savoia C, Battistoni A, Calvez V, Cesario V, Montefusco G, Filippini A. Microvascular alterations in hypertension and vascular aging. Curr Hypertens Rev. 2017;13(1):16-23.

26. Konukoglu D, Uzun H. Endothelial dysfunction and hypertension. Adv Exp Med Biol. 2017;956:511-40.

27. Pan $\mathrm{CH}$, Chen CJ, Shih CM, Wang MF, Wang JY, Wu CH. Oxidative stress-induced cellular senescence desensitizes cell growth and migration of vascular smooth muscle cells through down-regulation of platelet-derived growth factor receptor-beta. Aging. 2019;11:8085.

28. Shiels MS, Katki HA, Freedman ND, et al. Cigarette smoking and variations in systemic immune and inflammation markers. J Natl Cancer Inst. 2014;106(11):dju294.

Publisher's Note Springer Nature remains neutral with regard to jurisdictional claims in published maps and institutional affiliations. 